\title{
Effect of 5-HTTLPR on connectivity and topological properties of resting state EEG networks
}

\author{
G. Knyazev, E. Proshina, A. Savostyanov*, A. Bocharov \\ Institute of Physiology and Basic Medicine, Novosibirsk, Russia \\ *e-mail: Alexander.Savostyanov@gmail.com
}

Key words: serotonin transporter gene, promoter, genetic variants, magnetic resonance imaging, brain networks

Serotonin transporter is one of the most widely investigated genetic markers of individual variation in serotoninergic function. The promoter region of the serotonin transporter gene (5-HTTLPR) contains long (L) and short (S) variants with the latter one having reduced transcriptional efficiency. $\mathrm{S}$ allele has been found to increase the risk of depression and other mental health problems, but some evidence suggests that S-allele carriers outperform subjects carrying the long allele in an array of cognitive tasks. Functional magnetic resonance imaging studies demonstrate a heightened amygdala response to negative emotional stimuli and diminished connectivity among key areas involved in emotion regulation in $\mathrm{S}$ allele carriers. However, evidence linking this polymorphism with individual variation in electrophysiological properties of resting state brain networks is still very limited. This study investigated the effect of 5-HTTLPR polymorphism on EEG current source density, connectivity, and topological properties of resting state networks. As compared to L homozygotes, S-allele carriers showed lower current source density and connectivity in most frequency bands in areas overlapping with the default mode and emotion regulation regions. The analysis of graph-theoretical measures showed that as compared to L homozygotes, S-allele carriers have less optimal topological properties of brain networks in theta, but more optimal in alpha band. This dissociation may reflect predisposition to emotional disorders, which is inherent to $\mathrm{S}$ allele carriers, and, on the other hand, their superior functioning in some cognitive domains.

Acknowledgements: The study was supported by the Russian Science Foundation (RSF) under Grant No. 17-18-01019. 